\title{
Ultrawide Field Imaging in Retinal Diseases
}

\author{
Hidayet Erdöl*, Dilek Uzlu, Mehmet Kola and Nurettin Akyol \\ Department of Ophthalmology, Karadeniz Technical University, Turkey \\ *Corresponding author: Hidayet Erdöl, Department of Ophthalmology, Karadeniz Technical University, Turkey
}

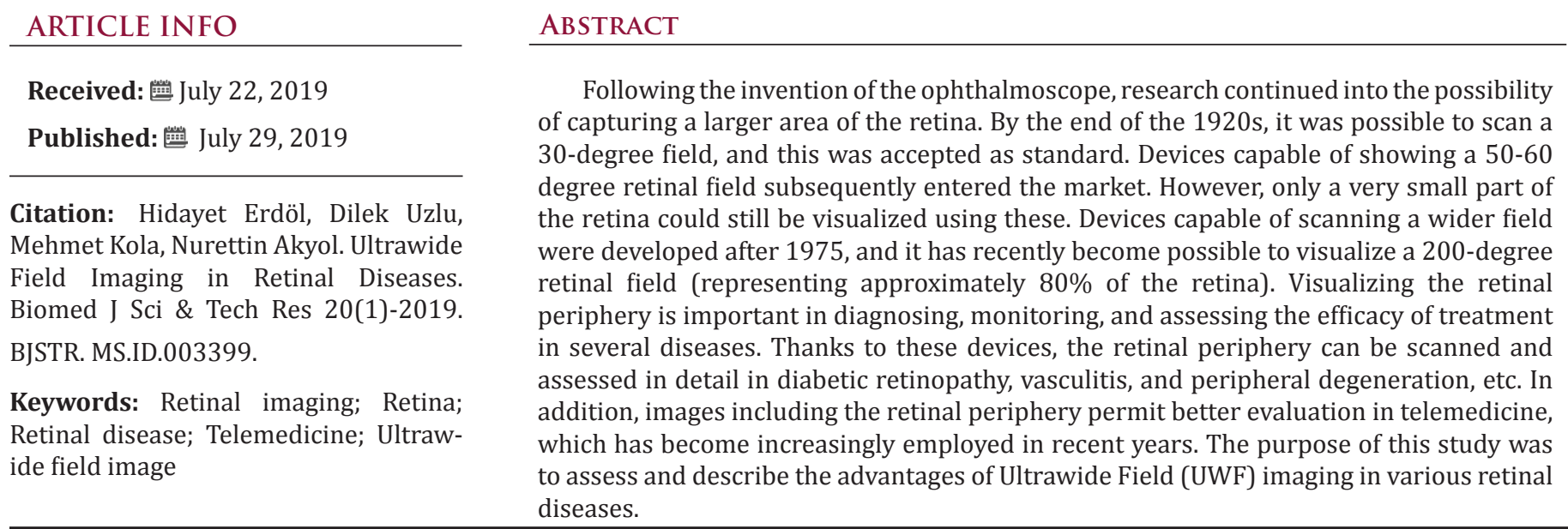

\section{Introduction}

\section{The Development of Imaging Devices}

Following the invention of the ophthalmoscope by Hermann von Helmholtz in 1851, the first fundus camera was placed on the market by Zeiss in 1926. The field provided by that device was approximately 20 degrees [1]. The field capable of being visualized subsequently increased to 30 degrees, and this became recognized as standard in retinal imaging. Stereophotographs consisting of Seven Standard Fields (7SF) showing an approximately 90-degree retinal field were developed in the ETDRS and recognized as the standard Ultrawide Field (UWF), and were used for classification in studies concerning Diabetic Retinopathy (DR) [2,3]. It is important to analyze the peripheral retina and document the findings obtained in order to evaluate the disease more accurately such as Diabetic Retinopathy (DR), venous occlusions, retinal and choroidal masses, uveitis (including vasculitis), peripheral retinal degeneration, retinal detachment, Retinopathy Of Prematurity (ROP) and tumors $[4,5]$. The first wide-angle camera system capable of also visualizing the equator was developed in 1975 by Pomerantzeff. This system is capable of capturing a 148-degree retinal field by means of a contact lens and scleral transillumination [6]. Retcam (130 degree) (Clarity Medical System, Inc., Pleasanton, CA, USA) entered the market in 1997 [7]. After Panorett-100 (Medibell Medical Vision Technologies Haifa, Israel) which is capable of capturing an approximately 100-degree retinal field with a contact lens and scleral illumination [8], Optos (Optos PLC, Dunfermline, UK) (200 degree) and the Heidelberg Spectralis (Heidelberg Engineering Inc., Heidelberg, Germany) (150 degree) devices entered the market $[9,10]$. After the development of wide-angle imaging systems, there have been some changes in the approach to retinal diseases. In this article, we aimed to emphasize the importance and properties of wide angle imaging in some retinal diseases.

\section{Common Areas of use of Ultrawide Field Imaging}

Diabetic Retinopathy: DR is the leading cause of vision loss in all age groups. Retinal imaging and evaluation of the photographs taken are of great importance in the monitoring of retinopathy. The course of pathologies can be objectively evaluated using color fundus photographs and fluorescein angiography [3]. Although DR affects the macula, it may cause serious pathologies in the retinal periphery. [11] showed that peripheral lesions missed at standard imaging can be detected using wide field imaging. In addition, they showed that retinopathy was more severe than indicated with standard imaging in $20 \%$ of cases. Price et al. [12] compared UWF 
scanning with ETDRS 7SF imaging and determined DR at more severe levels in $19 \%$ of cases [11]. Wessel et al. [13] showed the presence of ischemia and Neo-Vascularization (NV) that could not be captured in seven standard fields at FA in $10 \%$ of cases, but which could be visualized in the periphery with UWF. UWF imaging has come to occupy an important place in determining peripheral diabetic lesions in ophthalmological practice, and is important for improved evaluation of the disease. The Diabetic Retinopathy Research Network protocol AA study aims to assess DRP progression with a combination of FFA and peripheral findings on colored photographs, and the results are expected to be published in 2020 [14].

Retinal Vascular Diseases: Vascular occlusions are the second most common retinal pathologies after DR. Branch Retinal Vein Occlusion (BRVO) and Central Vein Retinal Occlusion (CRVO) are particularly common conditions. Ischemia, the most important factor affecting the prognosis of the disease, can accompany these pathologies, and the therapeutic approach varies depending on the degree of ischemia. UWF imaging offers a number of advantages over standard imaging in vascular pathologies. In particular, it permits determination of peripheral perfusion and analysis of the diffusion of ischemia in a single frame at FFA. Imaging performed by recording the retinal periphery has an important effect on the course of the disease and treatment planning [15]. Peripheral ischemia and NV in retinal vein obstructions can easily be shown with UWF imaging devices. Correlation has been shown between peripheral ischemia and macular edema in BRVO or hemicentral RVO [5]

Inflammatory Diseases of the Retina: Inflammatory diseases are known to affect both the posterior pole and the periphery of the retina. Peripheral pathologies can be clearly recorded using UWF scanning and all pathologies can be shown on a single frame. Sheathing, non-perfused fields and hyperfluorescent spots in vasculitis can all be revealed more clearly in this way. UWF scanning has been described as a particularly important tool in the diagnosis, monitoring and treatment of Behçet-related retinal vasculitis [16].

Retinopathy Of Prematurity: ROP is one of the important diseases seen in preterm infants. It is possible to show and capture wider visualization of retina with the devices capable capturing wide angle. In this way, vascular development in infants can be shown and monitored in a single frame. Therefore, potential medicolegal problems can be prevented in this way [17].

Peripheral Retinal Degenerations and Tumors: Various studies have reported that wide-field FFA and FAF imaging can facilitate the diagnosis and follow-up of these diseases. Another important issue is that peripheral retinal degeneration is common and some of these pathologies, such as lattice, snail track and white without pressure, can lead to tears in the retina [18]. The importance of UWF imaging in both primary and metastatic lesions (malignant melanoma, von Hippel Lindau, RPE adenoma, and metasta- sis) of the retina and choroid has been emphasized in many studies $[19,20]$.

\section{Retinal Detachment}

Another clinical condition requiring careful evaluation of the periphery of the retina is retinal degeneration and retinal detachment. UWF scanning permits a relatively greater width of image compared to ophthalmoscopy, together with easier visualization and documentation of existing holes in detachments, which generally assume the form of tears. In addition, several tears can be visualized simultaneously on UWF images [21]. Similarly, UWF images provide valuable information for assessing dimensions in retinal dialysis, since the extent of retinal detachment may vary [22]. UWF imaging systems will elicit more powerful data and permit more accurate assessment of the peripheral retina. One study reported that $100 \%$ of peripheral retinal tears were identified in 65 patients with acute posterior vitreous detachment using the Staurenghi lens system. However, the authors also reported that this method could not replace clinical examination [23].

\section{Age-Related Macular Degeneration}

Age-Related Macular Degeneration (AMD) may involve various lesions in the retinal periphery. Accompanying changes in the periphery can be better revealed and their characteristics better evaluated with FAF and colored images using UWF scanning. Witmer et al. [24] determined FAF anomalies in the periphery in $63.6 \%$ of patients with AMD, compared to $35.7 \%$ for the control group, the difference being statistically significant. They reported that characterization of different peripheral phenotypes might be important for the diagnosis and treatment of AMD subtypes. Madhusudhan et al. [25] recently reported an association between peripheral leakage shown at UWF and active neovascular AMD in some patients compared to eyes without active neovascular AMD.

\section{Conclusion}

In conclusion, wide-field imaging provides provide more comprehensive information about the retinal periphery, which is important for the screening, diagnosis, monitoring, and treatment of many vision-threatening eye diseases such as DR, ROP, central or branch vein obstructions, uveitis, and tumors. In particular, it provides more diagnostic information in telemedicine, which is now becoming increasingly widely employed, than does standard imaging. The short scanning time, lower storage, and the ability to show several pathologies in the same frame will assist the physician make more accurate decisions. In our opinion, UWF scanning systems will lead to new interpretations of many diseases in the soon future and will make a revision of telemedicine systems essential.

\section{References}

1. Salz DA, Witkin AJ (2015) Diabetic retinopathy update imaging in diabetic retinopathy. 22: 145-151.

2. Treatment Diabetic Retinopathy Study Research Group E (1991) Fundus photographic risk factors for progression of diabetic retinopathy ETDRS 
report number 12. Early treatment diabetic retinopathy study research group. Ophthalmology 98: 823-833.

3. ETDRS (1991) Grading DR from stereoscopic color fundus photographsAn extension of the modified airlie house classification - ETDRS 10 . Ophthalmology 98: 786-806.

4. Spaide RF (2011) Peripheral areas of nonperfusion in treated central retinal vein occlusion as imaged by wide-field fluorescein angiography. Retina 31: 829-837.

5. Prasad PS, Oliver SC, Coffee RE, Hubschman JP, Schwartz SD (2010) Ultra wide-field angiographic characteristics of branch retinal and hemicentral retinal vein occlusion. Ophthalmology 117: 780-784.

6. Pomerantzeff 0 (1975) Equator-plus camera. Invest Ophthalmol 14: 401-406.

7. Nakagawa TA, Skrinska R (2001) Improved documentation of retinal hemorrhages using a wide-field digital ophthalmic camera in patients who experienced abusive head trauma. Arch Pediatr Adolesc Med 155: $1149-1152$.

8. Shields CL, Materin M, Shields JA (2003) Panoramic imaging of the ocular fundus. Arch Ophthalmol 121: 1603-1607.

9. Pandya A, Friberg T, Eller A (2002) Optos non-mydriatic widefield Imaging vs. Clinical dilated fundus exam for retinal diagnosis and management. Invest Ophthalmol Vis Sci 43: 2860.

10. Staurenghi G, Viola F, Mainster MA, Graham RD, Harrington PG (2005) Scanning laser ophthalmoscopy and angiography with a wide-field contact lens system. Arch Ophthalmol 123: 244-252.

11. Silva PS, Cavallerano JD, Sun JK, Soliman AZ, Aiello LM, et al. (2013) Peripheral lesions identified by mydriatic ultrawide field imaging: Distribution and potential impact on diabetic retinopathy severity. Ophthalmology 120: 2587-2595.

12. Price LD, Au S, Chong NV (2015) Optomap ultrawide field imaging identifies additional retinal abnormalities in patients with diabetic retinopathy. Clin Ophthalmol 9: 527-531.

13. Wessel MM, Aaker GD, Parlitsis G, Cho M, D’Amico DJ, et al. (2012) Ultrawide-field angiography improves the detection and classification of diabetic retinopathy. Retina 32: 785-791.

14. Muqit MM, Marcellino GR, Henson DB, Young LB, Patton N, et al. (2013) Optos-guided pattern scan laser (Pascal)-targeted retinal

ISSN: 2574-1241

DOI: $10.26717 /$ BJSTR.2019.20.003399

Hidayet Erdöl. Biomed J Sci \& Tech Res

CC) This work is licensed under Creative

Submission Link: https://biomedres.us/submit-manuscript.php photocoagulation in proliferative diabetic retinopathy. Acta Ophthalmol 91: 251-258.

15. Manivannan A, Plskova J, Farrow A, Mckay S, Sharp PF, et al. (2005) Ultra-wide-field fluorescein angiography of the ocular fundus. Am J Ophthalmol 140: 525-527.

16. Mesquida M, Llorenc V, Fontenla JR, Navarro MJ, Adan A, et al. (2014) Use of ultra-wide-field retinal imaging in the management of active Behcet retinal vasculitis. Retina 34: 2121-2127.

17. Patel CK, Fung TH, Muqit MM, Mordant DJ, Brett J, et al. (2013) Noncontact ultra-widefield imaging of retinopathy of prematurity using the Optos dual wavelength scanning laser ophthalmoscope. Eye 27: 589596.

18. Kernt M, Schaller UC, Stumpf C, Ulbig MW, Kampik A, et al. (2010) Choroidal pigmented lesions imaged by ultra-wide-field scanning laser ophthalmoscopy with two laser wavelengths (Optomap). Clin Ophthalmol 4: 829-836.

19. Reznicek L, Stumpf C, Seidensticker F, Kampik A, Neubauer AS, et al. (2014) Role of wide-field autofluorescence imaging and scanning laser ophthalmoscopy in differentiation of choroidal pigmented lesions. Int J Ophthalmol 7: 697-703

20.Shah SP, Jain A, Coffee RE, McCannel TA (2009) Optos panoramic 200MATM ultrawide-field imaging of peripheral RPE adenoma. Semin Ophthalmol 24: 37-39.

21. Kornberg DL, Klufas MA, Yannuzzi NA, Orlin A, D’Amico DJ, et al. (2016) Clinical utility of ultra-widefield imaging with the optos optomap compared with indirect ophthalmoscopy in the setting of non-traumatic rhegmatogenous retinal detachment. Semin Ophthalmol 31: 505-512.

22. Patel M, Kiss S (2014) Ultra-wide-field fluorescein angiography in retinal disease. Curr Opin Ophthalmol 25: 213-220.

23. Witmer MT, Kiss S (2013) Wide-field imaging of the retina. Surv Ophthalmol 58: 143-154

24. Witmer MT, Kozbial A, DanielS, Kiss S (2012) Peripheral autofluorescence findings in age-related macular degeneration. Acta Ophthalmol 90: e428-e433.

25. Madhusudhan S, Beare N (2014) Wide-field fluorescein angiography in wet age-related macular degeneration. Sci World J 2014: 7.

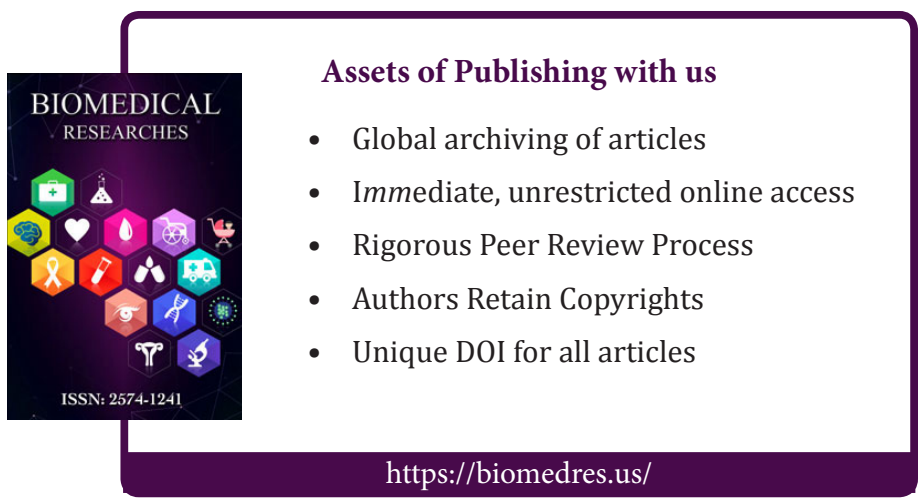

